\title{
Wi-Fi Sensing: Should Mobiles Sleep Longer As They Age?
}

\author{
Jaeseong Jeong ${ }^{\dagger}$, Yung $\mathrm{Yi}^{\dagger}$, Jeong-woo Cho ${ }^{\ddagger}$, Do Young Eun ${ }^{\S}$ and Song Chong ${ }^{\dagger}$ \\ jsjung@netsys.kaist.ac.kr, \{yiyung, songchong\}@kaist.edu, jwcho@kth.se, dyeun@ncsu.edu
}

\begin{abstract}
An essential condition precedent to the success of mobile applications based on Wi-Fi (e.g., iCloud) is an energyefficient Wi-Fi sensing. From a user's perspective, a good WiFi sensing policy should depend on both inter-AP arrival and contact duration time distributions. Prior work focuses on limited cases of those two distributions (e.g., exponential) or introduces heuristic approaches such as AI (Additive Increase). In this paper, we formulate a functional optimization problem on Wi-Fi sensing under general inter-AP and contact duration distributions, and propose how each user should sense Wi-Fi APs to strike a balance between energy efficiency and performance, depending on the users' mobility pattern. To that end, we derive an optimal condition which sheds insights into the aging property, the key feature required by efficient $\mathrm{Wi}-\mathrm{Fi}$ sensing polices. Guided by the analytical studies and the implications, we develop a new sensing algorithm, called WiSAG (Wi-Fi Sensing with AGing), which is demonstrated to outperform the existing sensing algorithms up to $34 \%$ through extensive trace-driven simulations using the real mobility traces gathered from smartphones.
\end{abstract}

\section{INTRODUCTION}

\section{A. Motivation}

The number of mobile users with smart phones/pads is rapidly increasing. Cisco reported that mobile data traffic grew 2.6 fold in 2010, and forecasts that it will increase 26-fold between 2010 and 2015 [1]. Smarter applications generating heavier traffic are expediting the scarcity of $3 \mathrm{G}$ capacity. $4 \mathrm{G}$, which has started to be deployed, seems to be only a temporary solution due to huge difference between traffic demands and available physical resources in the cellular system.

Leveraging Wi-Fi is an intriguing solution that has high potential in alleviating mobile data explosion. The very feature of shorter range communication of Wi-Fi than that of $3 \mathrm{G}$ or $4 \mathrm{G}$ on the unlicensed bands brings considerable efficiency in spatial frequency reuse. Wi-Fi $\mathrm{APs}^{1}$ also cost much less than cellular Base Stations [2], so that they can be deployed quickly as well as without heavy financial burden to operators and even users. In fact, Wi-Fi APs have already been installed around hotspots in many countries now. Very lately, researchers have started to examine the effect of Wi-Fi offloading from the theoretical and experimental perspectives [2]-[4]. For example, Lee et al. [2] showed that about $70 \%$ cellular data can be offloaded to Wi-Fi if users would tolerate two hour delayed data delivery. Wi-Fi is particularly useful for applications that

†: Department of Electrical Engineering, KAIST, South Korea. $\ddagger$ : School of Information and Communication Technology at KTH Royal Institute of Technology, Sweden. §: Department of Electrical and Computer Engineering, North Carolina State University, USA.

${ }^{1}$ We simply use 'AP' throughout this paper. periodically exploit the network, e.g., iCloud [5], Microsoft Pocket Outlook [6] and Urban Tomography System [7]. Wi-Fi connectivity can also provide the location information, whose economic value is huge [8].

Yet, not every user seems to welcome Wi-Fi. The survey by Devicescape [9] tells us that $64 \%$ of US consumers hit hot spots at least once a day, but some of them sometimes spend a day without Wi-Fi connections. From mobile users' perspective, one of the biggest concerns lies in quick battery discharge by Wi-Fi sensing [10]-[16]. It is reported [17] that $41 \%$ of iPhone $3 \mathrm{G}$ users and $15 \%$ of iPhone 4 users mention such a battery concern. Therefore, in order to maximally exploit WiFi's benefits, it is essential to relax users' attention to battery drainage by developing energy-efficient sensing schemes that sense scattered APs while sparingly using mobiles' batteries.

\section{B. Summary and Main Contributions}

In order to design the "best" Wi-Fi sensing scheme, we can gather that there exists a fundamental tradeoff between energy efficiency and performance: Sensing with less frequency results in bigger energy saving, but entails lower chances of data transmission through Wi-Fi. To strike a good balance, two key factors from a user's mobility pattern need to be carefully addressed: (i) how often users meet APs (referred to as inter$A P$ arrival time), and (ii) how long a user is in contact with an AP (called AP contact duration time).

Prior work [13], [18] studied optimal sensing intervals for only limited cases, e.g., exponential inter-AP arrivals and contact durations, in which periodic sensing interval is optimal. They also introduced heuristic algorithms such as AI [18] and WiFisense [13], both of which propose to increase the sensing intervals whenever they fail to detect an AP. However, the following questions still remain: (i) What is an optimal sensing policy for the users who do not have memoryless exponential inter-AP arrivals and contact duration distributions? (ii) When do the heuristic algorithms that increase sensing intervals for AP meeting failures work well? These questions are of significant importance, because, as discussed in Section IV, users have diverse mobility patterns, thus diverse distributions on inter-AP arrival and contact duration times.

To answer the questions above, we take a holistic approach by formulating a mathematical problem that captures a user's mobility pattern under general distributions of inter-AP arrival and contact duration time, and the tradeoff between energy consumption and contact loss. More formally, we adopt a functional optimization approach, where our objective is to 
TABLE I

KEY FINDINGS FOR SENSING INTERVAL. OUR NEW FINDINGS: bold-face

\begin{tabular}{|c||c|c|c|}
\hline \multirow{2}{*}{ Contact duration } & \multicolumn{3}{|c|}{ Inter-AP arrival (aging) } \\
\cline { 2 - 4 } & Negative & Constant & Positive \\
\hline \hline $\begin{array}{c}\text { Weibull } \\
\text { Generalized Pareto }\end{array}$ & increase & periodic [18] & decrease \\
\hline Exponential & increase & periodic [13], [18] & decrease \\
\hline
\end{tabular}

minimize a function of sensing process over time. By computing a necessary condition for optimality, which is also sufficient under mild cases, we first find that the key factor to how we should sense APs optimally is simply the aging property of an inter-AP arrival time, for a given AP contact duration distribution. The notion of aging property is from reliability theory [19], intuitively explained as follows: Consider an event that a mobile node has not been in contact with an AP until time $x$. We say that aging of the inter-AP arrival time, is positive (resp. negative), if when $x$ increases, the remaining time to meet an AP from $x$, stochastically decreases (resp. increases). Analysis through the aging concept extremely simplifies the understanding of the "best" sensing process, providing diverse practical implications. Our theoretical study reveals how sensing intervals should be chosen depending on a user's diverse mobility patterns measured by inter-AP arrival and contact duration distributions, as summarized in Table I. From the theory-driven implications, we develop a new sensing algorithm, called WiSAG (Wi-fi Sensing with AGing), which adaptively varies sensing intervals depending on the features of inter-AP arrival and contact duration times.

In order to study the distributions of inter-AP arrivals and contact durations of real users and evaluate the performance of WiSAG, we analyze Wi-Fi connectivity logs from the two different traces: (i) 84 3G/3GS iPhone users for 18 days where users are recruited from an iPhone user community in South Korea [2] and (ii) 60 students in KAIST campus students using Android smartphones for 14 days. Both traces reveal that a large fraction of participants have a negative aging property, which claim that their mobiles should sleep longer as the elapsed time from the last AP contact increases. This shows the case when the heuristic algorithms such as AI may work well. However, from our simulations, WiSAG outperforms even AI by up to about $25 \%$ on average, because better parameter selection, e.g., amount of increasing intervals, can be made in WiSAG than AI. We also observe the relatively regular AP contact patterns from several users who have a positive aging in their distributions. In this case, optimal sensing interval should decrease over time from our theoretical findings. Thus, the existing sensing algorithms [13], [18], which increases sensing intervals fail to achieve high performance, showing about $47 \%$ performance gap on average. The key performance improvement over existing algorithms lies in the ability that our theory-inspired algorithm, WiSAG provides a macroscopic guideline on sensing intervals, i.e., increase or decrease, and further proposes more exact amount of intervals.

\section{Related Work}

There have been many studies on energy-efficient Wi-Fi sensing [13]-[16], [18] based on the Wi-Fi sleep mode. Wi-
Fi sensing policies using surrounding information includes cellular fingerprint [14], Zigbee [15], accelerometer [13] and bluetooth logs [16], where the fact that the information is closely correlated to Wi-Fi AP contacts is exploited. However, utilizing those information incurs additional overheads, e.g., battery consumption for collecting the informations [10] and additional resource usage for maintaining and processing the information [14]. Despite the research results that those algorithms are efficient in sensing in several practical scenarios, there may exist some cases where (i) the available surrounding information is sparsely encountered, (ii) the additional overheads become a crucial part, (iii) the uncertainty of estimating an AP contact time is still high even with those surrounding information. Thus, considering the randomness of AP contact patterns without explicit support from surrounding information can also be an essential part of WiFi sensing. In fact, most of current smartphones do not use the surrounding informations in AP sensing but sense only on-demand [13] for saving energy. This paper provides key guidelines to choose sensing intervals and proposes an energy-efficient sensing algorithm under a random AP contact patterns, where such a "blind sensing" 2 is expected to still be important in smartphones.

\section{Model And OBJective}

\section{A. System Model}

Phases. Mobile nodes (or simply mobiles) move over time and intermittently meet Wi-Fi APs. We divide the entire time into a sequence of phases, where a phase corresponds to a time interval ranging from (i) the instant when a mobile node loses a Wi-Fi contact and to (ii) the end of the next Wi-Fi contact, as depicted in Fig. 1.

Inter-AP arrival and contact duration. Each phase is split into two intervals of when a mobile is not under Wi-Fi coverage, i.e., inter-AP arrival time, and when a mobile is under Wi-Fi coverage, i.e., AP contact duration time. We say that a mobile encounters an AP when it starts to be under the coverage of an AP. We also say that the mobile is associated with an AP, when a mobile is aware of being under the AP's coverage, and ready for data transmission at the average rate of $r_{w}$. Denote by $X$ and $Y$ the random variables of inter-AP arrival and contact duration times, respectively. Let $F_{X}(x)=\mathbb{P}[X<x]$ and $F_{Y}(y)=\mathbb{P}[Y<y]$ and we use $\bar{F}_{X}(x)=1-F_{X}(x)$ and $\bar{F}_{Y}(y)=1-F_{Y}(y)$. We assume that phases are independent, i.e., $X$ and $Y$ are i.i.d. across phases, which enables us to focus on a single phase. Due to the recent papers [2], [20], [21], it has been shown that humans' movement patterns are highly regular. Thus, we assume that each mobile knows its distributions of inter-AP arrival and contact duration times.

Sensing. As depicted in Fig. 1, let $T_{0}$ be the starting time of a phase, and a mobile senses APs at times $T_{1}, T_{2}, \cdots$. A sequence $\left(T_{k}\right)_{k=1}^{\infty}$ is randomly generated by a sensing process $N(t)$ which is a non-homogeneous Poisson process with rate $n(t)$. Since $n(t)$ may take the form of a pulse

\footnotetext{
${ }^{2}$ In this paper, we define "blind sensing" as a sensing scheme which does not use any of surrounding information.
} 


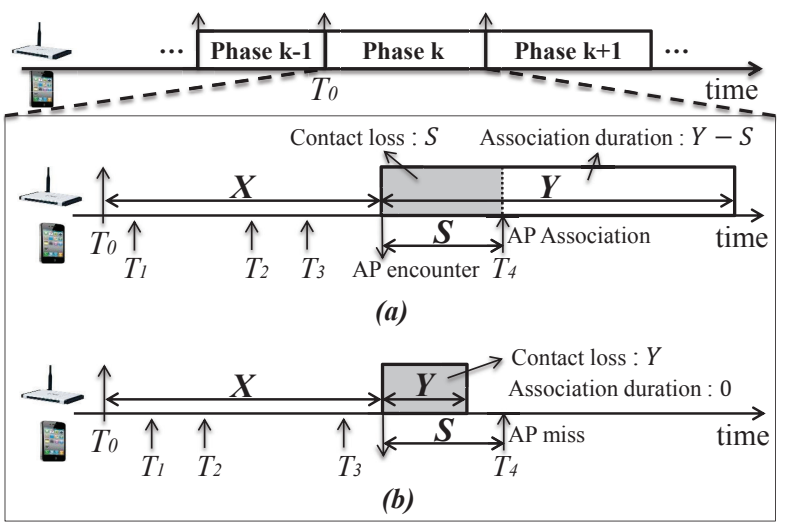

Fig. 1. An illustration of the model. (a) When AP contact duration $Y$ is larger than $S$, a mobile successfully detects an AP. (b) When AP contact duration $Y$ is smaller than $S$, a mobile node fails to detect it. Shaded areas denote the contact loss time $l(S)$.

train of Dirac delta measures, called Dirac comb, $N(t)$ can model deterministic sensing as well as stochastic one. Let $c_{s}$ be the sensing cost, i.e., power consumption per one sensing operation. We assume that mobiles stop sensing after being associated. We also assume that the mobile is able to detect the end of each contact immediately after being outside of Wi-Fi coverage. This assumption is reasonable, since the connection loss can be quickly detected if some data transmission is in progress. For the case where a mobile misses a contact due to its large sensing interval, we will discuss later in Section III-E.

Loss time. Note that a mobile can be associated with an AP some time after encountering the AP (time $T_{4}$ in Fig. 1(a)). Let a random variable $S$ be the elapsed time until the next sensing since encountering. We are interested in the loss time that quantifies the duration that a mobile node misses the chance to use an AP. In Fig. 1(a), a mobile senses while being in the AP's coverage, where the loss time is $S$. However, in Fig. 1(b), the mobile moves fast, resulting in missing the AP contact, in which case the loss time is $Y$. Thus, the average loss time $l(S)$ (with respect to the contact duration $Y$ ) is:

$$
l(S)=\mathbb{E}_{Y}[\min \{Y, S\}] .
$$

\section{B. Problem Formulation}

Sensing with less frequency saves larger energy, which in turn increases the chance to miss Wi-Fi contacts, hence lower performance. We set a functional optimization problem with a single objective that combines energy efficiency with performance in a linear fashion, where one metric is treated as a penalty term. To be more specific, the optimization problem is to minimize the average sensing cost, linearly penalized by missed Wi-Fi contacts, over all feasible sensing policy $N(t)$. By doing so, two conflicting objectives of energyefficiency and performance, can be appropriately considered in the formulation.

\section{Minimize sensing cost penalized by lost Wi-Fi contacts}

$$
\min _{(n(t))_{t=0}^{\infty}}\left\{c_{s} \mathbb{E}[N(X)]+\gamma r_{w} \mathbb{E}[l(S)]\right\},
$$

(OPT)

where $\gamma$ is a penalty weight for the contact loss.
Recall that $\mathbb{E}[N(X)]$ is the average number of sensings before the mobile encounters an $\mathrm{AP}$, and $r_{w} \mathbb{E}[l(S)]$ corresponds to the average volume of data that cannot be transmitted due to the "lazy" association to the AP.

Then, our main interest is now centered on minimizing (OPT) by generating the random sequence $\left(T_{k}\right)_{k=0}^{\infty}$ (or choosing $n(t)$ for every $t \in(0, \infty)$ ), and thereby determining the non-homogeneous Poisson process $N(t)$, where $F_{X}(\cdot)$ and $F_{Y}(\cdot)$ are given. We first epitomize the notion of aging which will be frequently used in many parts of the discussion throughout this paper.

\section{Preliminary: Aging}

We define a failure rate [19] that quantifies the probability that a r.v. $X$ (e.g., inter-AP arrival time in our case) is some value, say $t$, on the condition that $X \geq t$ (e.g., a mobile does not meet an AP until time $t$ ).

Definition 1 Consider a r.v. $X$ with the PDF and CDF of $f_{X}(t)$ and $F_{X}(t)$, respectively. The failure rate $r_{X}(t)$ of $X$ is: $r_{X}(t) \triangleq \frac{f_{X}(t)}{F_{X}(t)}$, for the age $t$ such that $\bar{F}_{X}(t)>0$.

We assume that $r_{X}(t)$ is a real-valued, differentiable function. $r_{X}(t)$ is said to be Increasing Failure Rate (IFR) when $r_{X}(t)$ is strictly increasing, i.e., $r_{X}^{\prime}(t)>0, \forall t$. Likewise, $r_{X}(t)$ is called Decreasing Failure Rate (DFR) if $r_{X}^{\prime}(t)<0, \forall t$. Suppose that a r.v. $X$ follows a Weibull distribution, $F_{X}(t)=$ $1-\exp \left(-(t / \beta)^{\alpha}\right)$. Then the corresponding failure rate $r_{X}(t)$ is DFR (resp. IFR) for $0<\alpha<1$ (resp. $\alpha>1$ ).

Consider a r.v. $X$ with failure rate $r_{X}(t)$. We say that $X$ has positive aging if $r_{X}(t)$ is IFR. Similarly, $X$ is said to have negative aging if $r_{X}(t)$ is DFR. When $r_{X}^{\prime}(t)=0, X$ is said to have constant aging. The aging property can be understood by a residual time $X_{t}$ whose CCDF (Complementary CDF) is given by $\mathbb{P}\left[X_{t}>x\right]=\mathbb{P}[X-t>x \mid X>t]$. It is easy to see that when $r_{X}(t)$ is DFR, $X_{t}$ stochastically increases with time $t$. To interpret it in our context, on the condition that a mobile has no contact with an AP until $t$, the remaining time until encountering an AP from $t$ stochastically increases with $t$. For the case of IFR, a similar interpretation can be stated. This aging concept has recently been used in [22] which proves that this memory structure arises from general mobility patterns and can be used for a better design of mobile wireless networks.

\section{SOlutions AND Algorithm}

In this section, we first present the technical challenges of (OPT), by introducing the examples for two types of sensing policies. Then, we provide reasonable approximations of (OPT), and develop a modified objective function which allows mathematical tractability. Then, we derive the conditions for optimality, followed by the practical implications into a good Wi-Fi sensing algorithm.

\section{A. Challenges}

1) Homogeneous Poisson sensing: Let us first consider a simple case when sensing is performed following a homogeneous Poisson process in order to clearly see the tradeoff between energy efficiency and performance as well as to have 
a taste of challenges residing in our optimization problem. Consider a homogeneous Poisson process with rate $\mu$ and an AP contact duration time $Y$. Suppose $Y$ can be expressed in terms of another positive r.v. $A$ such that $\mathbb{P}[Y>y]=\mathbb{E}_{A}\left[\mathrm{e}^{-A y}\right] .^{3}$ Putting $c_{s}=\gamma=r_{w}=1$, (OPT) can be rearranged as:

$$
(\mathrm{OPT})=\min _{\mu}\{\mu \mathbb{E}[X]+\mathbb{E}[\min (Y, S)]\}
$$

where $S$ is an exponential r.v. (because of homogenous Poisson sensing), and

$$
\begin{aligned}
\mathbb{E} & {[\min (Y, S)]=\int_{0}^{\infty} \mathbb{P}[Y>x] \mathbb{P}[S>x] \mathrm{d} x } \\
= & \int_{0}^{\infty} e^{-\mu x} \mathbb{E}_{A}\left[e^{-A x}\right] \mathrm{d} x=\mathbb{E}_{A}\left[\frac{1}{\mu+A}\right] .
\end{aligned}
$$

Then it follows from (3) that (2) becomes:

$$
\min _{\mu}\left\{\mathbb{E}_{A}\left[\mu \mathbb{E}[X]+\frac{1}{\mu+A}\right]\right\}
$$

which clearly shows the tradeoff: as $\mu$ increases (i.e., sensing with higher frequency), the energy consumption term $\mu \mathbb{E}[X]$ increases, whereas the contact loss term $1 /(\mu+A)$ decreases.

Note that (4) is invariant with respect to the distribution of $X$ except for $\mathbb{E}[X]$, which is due to the restriction of our focus to homogeneous Poisson sensing processes. To put it another way, in order to find a more optimal sensing, it is inevitable that the sensing policy is modeled by a more general stochastic process, i.e., non-homogeneous Poisson processes, which lends itself to adaptation to distributional properties of $X$ and $A$, along with $\mathbb{E}[X]$.

2) Deterministic sensing: We now consider another class of sensing processes: deterministic sensing. Recall that this case can be regarded as non-homogeneous Poisson processes, where the rate $n(t)$ is a Dirac comb (See Section II-A). Then, it is not hard to see that computing the optimal solution of (OPT) is equivalent to solving the following problem, expressed in terms of distribution $F_{X}(\cdot)$ of the inter-AP arrival time $X$ :

$$
(\mathrm{OPT})=\min _{\left(T_{k}\right)_{k=0}^{\infty}} \sum_{k=1}^{\infty} \int_{T_{k-1}}^{T_{k}}\left\{c_{s} k+\gamma r_{w} l\left(T_{k}-t\right)\right\} \mathrm{d} F_{X}(t)
$$

Yet, solving (5) is significantly challenging due to the facts:

(i) Analytical solutions are hard to obtain, because each objective is a function of an infinite sequence $\left(T_{k}\right)_{k=0}^{\infty}$. On top of that, even for well-known distributions of $X$, there exists a complex coupling between a nonlinear function $l(\cdot)$ and the PDF $\mathrm{d} F_{X}(\cdot)$ inside the integral.

(ii) Computing the solutions numerically is also challenging, due to a large search space generated by an infinite number of possible combinations [23].

A similar problem for deterministic sensing processes has been studied in reliability theory, referred to as inspection problem [24], only when the contact duration time $Y$ is infinite (w.p. 1). Barlow et al. [24] developed a recurrence formula,

${ }^{3}$ In this case, $Y$ is called a completely monotone (CM) distribution. We use a $\mathrm{CM}$ distribution only to better illustrate the simplistic form of the tradeoff. Many types of distribution including Weibull are known to belong to CM class. instead of a fully analytical solution, only in the limited case. ${ }^{4}$ It still remains open as to how to solve the problem for general distributions.

\section{B. Approximations}

Obtaining the analytical form of $\mathbb{E}[l(S)]=\mathbb{E}[\mathbb{E}[l(S) \mid X]]$, entails two key obstacles: (i) the analytical form of $S$ 's distribution (conditioned on $X$ ), given by:

$$
\mathbb{P}[S>s \mid X=t]=\exp \left(-\int_{t}^{t+s} n(u) \mathrm{d} u\right),
$$

is difficult to compute, and (ii) non-linear loss time function $l(\cdot)$ is involved there. Thus, to get a tractable form of the objective function, we make two approximations, summarized in what follows.

First, we use an upper bound of $\mathbb{E}[\mathbb{E}[l(S) \mid X]]$. Note that it is easy to show that $l(s)$ is concave in $s$, verified by:

$$
l(s)=\int_{0}^{\infty} \mathbb{P}[\min \{Y, s\}>y] \mathrm{d} y=\int_{0}^{s} \mathbb{P}[Y>y] \mathrm{d} y,
$$

whereupon we have $l^{\prime \prime}(s)=-f_{Y}(s)<0$. It follows from Jensen's inequality that $\mathbb{E}[\mathbb{E}[l(S) \mid X]] \leq \mathbb{E}[l(\mathbb{E}[S \mid X])]$. Manipulating $\mathbb{E}[S \mid X]$ becomes much more tractable due to the absence of $l(\cdot)$.

Now, for the computation of the new target $\mathbb{E}[l(\mathbb{E}[S \mid X])]$, it is required to get the analytical form of $\mathbb{E}[S \mid X=t]$ for $t>0$. However, it is still challenging to compute, because an integral with $n(t)$ is an exponent of the function $\exp (\cdot)$ in (6). To tackle this problem which is of vital importance to the optimization (OPT), we only assume that the following two quantities vary smoothly with $k$ :

$$
\mathbb{E}\left[T_{k}-T_{k-1}\right], \quad \sigma_{T_{k}-T_{k-1}}^{2}
$$

for $k \geq 1$, where the second term is the variance of $T_{k}-T_{k-1}$. This assumption implies that when a mobile conducts sensing, the average and variance of the next sensing time should be slowly-varying with each sensing. It should be remarked that the assumption specifies nothing else but the tendency of the first two moments, so that $n(t)$ is still allowed to vary with time $t$. Recall that our target is to compute $\mathbb{E}[S \mid X=t]$, where $S=T(t)-t$ and $T(t)$ is the next sensing time at $t$, i.e., $T(t) \triangleq$ $\min _{k}\left\{T_{k} \mid T_{k}>t\right\}$. To simplify exposition, we first adopt

$$
I(t) \triangleq T_{k}-T_{k-1}, \quad \text { such that } T_{k}>t \geq T_{k-1} .
$$

The practical value of this slowly-varying moment assumption lies in that it enables us to get a manipulative form of $\mathbb{E}[S \mid X=t]$ which is the mean residual time of a point process at arbitrary time $t$. Note the arbitrary time $t$ is more likely to fall in larger sensing intervals around $t$. Thus it follows from the Palm inversion formula [26, Th. 7.3.1]:

$$
\Lambda(t) \triangleq \mathbb{E}[S \mid X=t]=\frac{1}{2}\left(\mathbb{E}[I(t)]+\frac{\sigma_{I(t)}^{2}}{\mathbb{E}[I(t)]}\right),
$$

${ }^{4}$ when $f_{X}(t)$ is $P F_{2}$ (Pólya frequency function of order 2). As discussed in [25, Remark 2.2], if the failure rate function $r_{X}(t)$ is DFR, $f_{X}(t)$ does not belong to the class of $P F_{2}$. Note that DFR $X$ has been largely seen in the real mobility traces (see Section IV for details). 
also known as Feller's paradox. To summarize, $\mathbb{E}[l(\mathbb{E}[S \mid X])]$ can be further approximated when the first two moments of inter-sensing times do not vary much with each sensing event.

Approximating the original contact loss time $\mathbb{E}[l(S)]$ with $\mathbb{E}[l(\mathbb{E}[S \mid X])]$, and plugging (8) into (OPT), and the original optimization objective can be rearranged as

$$
\begin{aligned}
& \min _{(n(t))_{t=0}^{\infty}} c_{s} \mathbb{E}\left[\int_{0}^{X} n(\tau) \mathrm{d} \tau\right]+\gamma r_{w} \mathbb{E}[l(\Lambda(X))] \\
= & \min _{(n(t))_{t=0}^{\infty}} \int_{0}^{\infty}\left\{c_{s} \int_{0}^{t} n(\tau) \mathrm{d} \tau f_{X}(t)+\gamma r_{w} l(\Lambda(t)) f_{X}(t)\right\} \mathrm{d} t \\
= & \min _{(n(t))_{t=0}^{\infty}} \int_{0}^{\infty} c_{s} n(t) \bar{F}_{X}(t)+\gamma r_{w} l(\Lambda(t)) f_{X}(t) \mathrm{d} t
\end{aligned}
$$

where $f_{X}(\cdot)$ is the density function of the inter-AP arrivals.

Here we can see that the first term inside the integral of (9) is still a function of $n(t)$, which can be further simplified from the fact that replacing the instantaneous rate $n(t)$ with its short-term average rate $1 / \mathbb{E}[I(t)]$ does not make significant difference to (9) if $\bar{F}_{X}(t)$ inside its integral is a well-defined smooth function. Finally, we present the modified objective approximating the original optimization objective, expressed by $X, 1 / \mathbb{E}[I(t)]$, and $\sigma_{I(t)}^{2}$ :

$$
\min _{(I(t))_{t=0}^{\infty}} \int_{0}^{\infty} \frac{c_{s}}{\mathbb{E}[I(t)]} \bar{F}_{X}(t)+\gamma r_{w} l(\Lambda(t)) f_{X}(t) \mathrm{d} t,
$$

$(\mathrm{xOPT})$

\section{Optimality Conditions}

The optimal sensing sequence from (xOPT) is computed by controlling the following two: (i) the expectation $\mathbb{E}[I(t)]$ and (ii) the variance $\sigma_{I(t)}^{2}$. Since the mean $\mathbb{E}[I(t)]$ and the variance $\sigma_{I(t)}^{2}$ are independent, we can freely adjust the variance while keeping the same mean of inter-sensing time. Note that for a fixed $\mathbb{E}[I(t)]$, (xOPT) increases with $\sigma_{I(t)}^{2}$, because the loss function $l(\Lambda(t))$ increases with $\Lambda(t)$. Thus, given $\mathbb{E}[I(t)]$, we first search the space of the sensing process $n(t)$ that minimizes the variance. It is not hard to see that a deterministic sensing process achieves the smallest variance, which is zero. This observation further simplifies the optimization (xOPT), where it suffices to solve (xOPT) over the space of deterministic sequences of sensing intervals with rate $n(t)$. Putting $\sigma_{I(t)}^{2}=0$, $\Lambda(t)$ in (8) becomes $\mathbb{E}[I(t)] / 2$. The objective function is then expressed as a functional $A[I]$ :

$$
A[I] \triangleq \int_{0}^{\infty}\left\{\frac{c_{s}}{I(t)} \bar{F}_{X}(t)+\gamma r_{w} l\left(\frac{I(t)}{2}\right) f_{X}(t)\right\} \mathrm{d} t .
$$

Note here that we need to determine only $\mathbb{E}[I(t)]=I(t)$ $(I(t)$ : the length of sensing interval containing $t)$ where the the equality holds because $I(t)$ is no longer random. To put it another way, we have now demonstrated that optimal WiFi sensing algorithms should be deterministic. As compared with (5), the deterministic formulation (10) has been properly justified and, thanks to its simplistic integral form, it is amenable to functional analysis which is applied to yield the following theorem. We apply the calculus of variations [27] to
$A[I]$ with the objective of finding an optimal $I(t)$, which leads to Theorem 1 presenting necessary and sufficient conditions for optimality. Denote by $\left(I^{\star}(t)\right)_{t=0}^{\infty}$ an optimal sensing interval function that minimizes $A[I]$.

\section{Theorem 1 (Optimality condition)}

(i) Necessity: (Recall that $r_{X}(t)$ is the failure rate of $X$ )

$$
\left(I^{\star}(t)\right)^{2} \bar{F}_{Y}\left(\frac{I^{\star}(t)}{2}\right)=\frac{2 c_{s}}{\gamma r_{w} r_{X}(t)},
$$

(ii) Sufficiency: (11) is also sufficient under the following condition:

$$
\bar{F}_{Y}\left(\frac{I^{\star}(t)}{2}\right)>\frac{I^{\star}(t)}{4} f_{Y}\left(\frac{I^{\star}(t)}{2}\right)
$$

Proof: To simplify the exposition, we first denote the reciprocal of $I(t)$ by: $\lambda(t) \triangleq 1 / I(t)$.

(i) Necessity. Let $F(\lambda(t))$ be the functional in (xOPT), i.e.,

$$
F(\lambda(t))=\int_{0}^{\infty}\left\{c_{s} \lambda(t) \bar{F}_{X}(t)+\gamma r_{w} l\left(\frac{1}{2 \lambda(t)}\right) f_{X}(t)\right\} \mathrm{d} t .
$$

From the functional derivative techniques in [27], [28], by differentiating the functional $F$ w.r.t. $\lambda(t)$ and setting it to zero, we obtain

$$
\begin{aligned}
\frac{\partial F}{\partial \lambda(t)} & =\frac{\partial}{\partial \lambda}\left\{c_{s} \lambda(t) \bar{F}_{X}(t)+\gamma r_{w} l\left(\frac{1}{2 \lambda(t)}\right) f_{X}(t)\right\} \\
& =c_{s} \bar{F}_{X}(t)-\gamma r_{w} \frac{f_{X}(t)}{2 \lambda(t)^{2}} l^{\prime}\left(\frac{1}{2 \lambda(t)}\right)=0 .
\end{aligned}
$$

By noting that $r_{X}(t)=f_{X}(t) / \bar{F}_{X}(t), l^{\prime}(x)=\bar{F}_{Y}(t)$ from (7), and $\lambda(t)=1 / I(t)$, (11) follows from (13).

(ii) Sufficiency. The necessary condition in (11) is also sufficient if the functional $F$ is convex [29], i.e.,

$$
\alpha F(\lambda(t))+(1-\alpha) F(\theta(t)) \geq F(\alpha \lambda(t)+(1-\alpha) \theta(t))
$$

for any two functions $\lambda(t), \theta(t)$ and $\alpha \in(0,1)$. This is equivalent to

$$
\begin{array}{r}
\int_{0}^{\infty}\left\{\alpha l\left(\frac{1}{2 \lambda(t)}\right)+(1-\alpha) l\left(\frac{1}{2 \theta(t)}\right)\right\} f_{X}(t) \mathrm{d} t \\
\geq \int_{0}^{\infty}\left\{l\left(\frac{1}{2(\alpha \lambda(t)+(1-\alpha) \theta(t)}\right)\right\} f_{X}(t) \mathrm{d} t,
\end{array}
$$

which holds if and only if $h(x):=l(1 / 2 x)$ is convex in $x>0$. Now, the condition $h^{\prime \prime}(x)>0$ reads as

$$
\frac{\mathrm{d}^{2}}{\mathrm{~d} x^{2}} h(x)=\frac{1}{x^{3}}\left[\bar{F}_{Y}\left(\frac{1}{2 x}\right)-\frac{1}{4 x} f_{Y}\left(\frac{1}{2 x}\right)\right]>0 .
$$

By replacing $x$ with $\lambda(t)$ and setting $1 / \lambda(t)=I(t)$, we get (12). This completes the proof.

As expected, Theorem 1 states that the optimal sensing sequence $I^{\star}(t)$ highly depends on the distributions of both inter-AP arrival and contact duration times, $X$ and $Y$. In the next subsection, we will explain that the sufficient condition in (12) is highly likely to be satisfied in practice, implying that the condition (11) is nearly necessary and sufficient for optimality. This motivates us to propose a novel sensing algorithm driven by (11) in Section III-C. 


\section{Mildness of Sufficient Condition (12)}

Now, we investigate the sufficiency region of the sensing interval $I(t)$. Since the sufficient condition (12) depends only on the distribution of contact duration time $Y$, we divide into four representative cases in terms of the distribution of $Y$. Throughout this section, we denote by $r_{Y}(t)$ the failure rate of the contact duration time $Y$, hence the sufficient condition (12) can be re-written as $\frac{4}{I(t)}-r_{Y}\left(\frac{I(t)}{2}\right)>0$, where $r_{Y}(t)=$ $f_{Y}(t) / \bar{F}_{Y}(t)$. For notational simplicity, we drop the superscript $\star$ in $I^{\star}(t)$ in the rest of this section, unless confusion arises.

(a) Heavy-tailed $Y$ in Generalized Pareto: When $Y$ follows a Generalized Pareto distribution with heavy-tail, we have $\mathbb{P}[Y>y] \sim\left(1+\frac{\xi y}{\sigma}\right)^{-\frac{1}{\xi}}$ with a shape parameter $\xi>\frac{1}{2}$ and a scale parameter $\sigma>0$. The term $\frac{4}{I(t)}-r_{Y}\left(\frac{I(t)}{2}\right)$ is always positive since $r_{Y}\left(\frac{I(t)}{2}\right)=\frac{2 / \xi}{2 \sigma / \xi+I(t)}$ is always less than $\frac{4}{I(t)}$, meaning that the interval sequence always be within the sufficiency region over time $t$. Note that the actual lengths of the increasing intervals can be numerically computed by solving the optimality condition (11) for the given distribution of inter-AP arrival time $X$ and contact duration time $Y$.

(b) Not Heavy-tailed $Y$ in Generalized Pareto: When $Y$ is Generalized Pareto r.v and not heavy-tailed, we have $\mathbb{P}[Y>$ $y] \sim\left(1+\frac{\xi y}{\sigma}\right)^{-\frac{1}{\xi}}$ with $\xi<\frac{1}{2}, \sigma>0$. Then, (12) becomes :

$$
\frac{4}{I(t)}-\frac{1}{\sigma+\frac{\xi I(t)}{2}}>0 \Leftrightarrow I(t)<\frac{4 \sigma}{1-2 \xi}=\frac{4 \mathbb{E}[Y](1-\xi)}{1-2 \xi}
$$

Shape parameter $\xi$ s measured from our trace in Section IV-B are greater than -0.5 , meaning that the intervals satisfy the sufficient condition when $I(t)<\zeta \mathbb{E}[Y], \zeta \in(3, \infty)$.

(c) Weibull $Y$ : When $Y$ follows a Weibull distribution, we have $\mathbb{P}[Y>y]=\exp \left\{-(y / \mu)^{\beta}\right\}$. For a Weibull distribution with the shape parameter $\beta$ and the scale parameter $\mu$, the condition for increasing interval is given by:

$$
\begin{aligned}
\frac{4}{I(t)}-\frac{\beta I(t)^{\beta-1}}{\mu^{\beta} 2^{\beta-1}}>0 & \Leftrightarrow \\
I(t) & <\frac{2^{1+1 / \beta} \mu}{\beta^{1 / \beta}}=\frac{2^{1+1 / \beta} \mathbb{E}[Y]}{\beta^{1 / \beta} \Gamma(1+1 / \beta)}
\end{aligned}
$$

The $\beta$ s of $Y$ from our trace range over the interval $[0.3,2]$. By rewriting (15) for such a range of $\beta$, the sufficiency interval region is $I(t)<\zeta \mathbb{E}[Y], \zeta \in[2.25,120]$.

(d) Exponential $Y$ : Note that a Weibull r.v. with $\beta=1$ is exponential. Thus, the condition for increasing intervals follows from (15), given by: $I(t)<4 \mathbb{E}[Y]$. This again means that the optimality holds unless the sensing interval exceeds $4 \mathbb{E}[Y]$.

The sufficiency condition (12) states that the optimal sensing intervals are not significantly large, compared to the AP contact duration time $Y$, whose mildness can be checked by measuring the distribution of $Y$ from real traces. According to the measurement, the optimal sensing interval is highly likely within the sufficiency region because 1) the penalty term from the contact loss in our optimization problem makes the interval scale smaller compared to $\mathbb{E}[Y]$ and 2) measured $\mathbb{E}[Y]$ is more than 2 times of measured $\mathbb{E}[X][2]$.

\section{E. WiSAG : Theory-Inspired Sensing Algorithm}

We now develop a Wi-Fi sensing algorithm, called WiSAG (Wi-fi Sensing with AGing), motivated and inspired by analytical findings in Theorem 1.

\section{WiSAG (Wi-fi Sensing with AGing)}

Preprocessing: A mobile computes a sensing interval function $I(t)$ according to (11), using the inputs of the distributions of inter-AP arrival and contact duration, $F_{X}(\cdot)$ and $F_{Y}(\cdot)$.

\section{At each phase $p$ :}

\section{Initialization.}

Set $T_{0}=0$, and $T_{1}=I\left(T_{0}\right)$.

Set the clock $t=0$ and the counter $k=1$, and run the clock.

2. If $t==T_{k}$,

Sense Wi-Fi

If no AP is sensed and associated,

Set $T_{k+1}=T_{k}+I\left(T_{k}\right)$ and $k=k+1$

Else communicate with the associated AP until the AP connection is lost and then end the phase $p$

\section{End If}

End If

A few remarks are in order. First, we assume that the distributions of $X$ and $Y$ are given to a user. This requires a mobile user to have a reasonable amount of training time. Although the detailed algorithm for training is beyond the scope of this paper, we can employ the training methods for other similar statistics, e.g., visiting patterns to a specific location such as office or home, used in other researches [20], [21]. Second, a practical algorithm like WiSAG, is unable to know whether the user misses an AP due to fast mobility or not, which differs from the model used in the analysis. In such a case, in the model, a new phase is assumed to start, yet in practice, the user is still in the old phase, and keeps increasing (or decreasing) the sensing intervals. We later show that Section IV demonstrates that such a difference is minor.

We now present the increase/decrease of optimal sensing intervals (as in WiSAG) in relation to the notion of aging of the inter-AP arrival time $X$. To that end, consider three cases when $X$ is negative, constant, and positive aging (i.e., $r^{\prime}(t)<0=$ $0,>0$, respectively). The trends are summarized as: Optimal sensing intervals $I(t)$ should increase, periodic, and decrease, if the distribution of $X$ has negative, constant, and positive aging, respectively, as stated in Table I in Section I.

To understand why, by rearranging the optimality condition (11) with $r_{X}(t)$ and $r_{Y}(t)$, we get:

$$
I(t)^{2} \exp \left(-\int_{0}^{I(t) / 2} r_{Y}(x) d x\right)=\frac{2 c_{s}}{\gamma r_{w} r_{X}(t)} .
$$

Taking $\log$ on both sides of (16) and differentiating w.r.t. $t$, we have the following:

$$
\frac{I^{\prime}(t)}{2}\left\{\frac{4}{I(t)}-r_{Y}\left(\frac{I(t)}{2}\right)\right\}=-\frac{r_{X}^{\prime}(t)}{r_{X}(t)}
$$

Once the failure rates of $X$ and $Y$ are given, (17) enlightens us upon the sign of $I^{\prime}(t)$. This further generates the conditions 


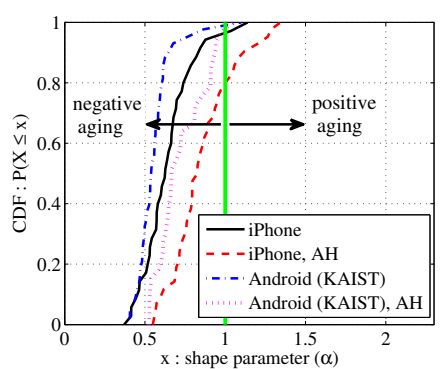

(a) Weibull, IAT

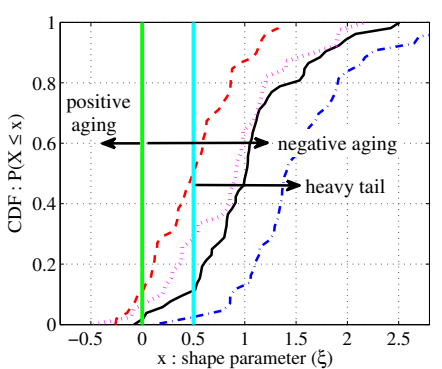

(b) Generalized Pareto, IAT

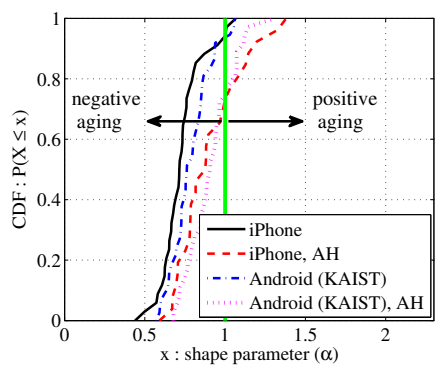

(c) Weibull, CDT

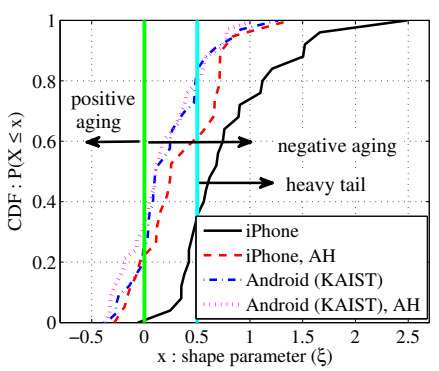

(d) Generalized Pareto, CDT

Fig. 2. Distribution of shape parameter $\alpha$ and $\xi$ in IAT and CDT of each individual in the traces.; (a)(b) CDFs of parameter $\alpha$ and $\xi$ of inter-AP arrival time (IAT) distributions of users identified as experiencing Weibull and Generalized Pareto IATs.; (c)(d) CDFs of parameter $\alpha$ and $\xi$ of contact duration time (CDT) distributions of users identified as having Weibull and Generalized Pareto CDTs.

for the signs of $I^{\prime}(t)$ and $\frac{4}{I(t)}-r_{Y}\left(\frac{I(t)}{2}\right)$, which provide the information on when the sensing intervals should increase or decrease, as elaborated shortly. We can easily check that $r_{X}(t)>0$ and the sufficient condition in (12) is exactly equal to $\frac{4}{I(t)}-r_{Y}\left(\frac{I(t)}{2}\right)>0$ in (17). Therefore, whether optimal sensing intervals should increase or decrease upon the failure of detecting an AP (i.e., the sign of $I^{\prime}(t)$ ) depends only on the sign of $r_{X}^{\prime}(t)$ which directly represents the aging property of inter-AP arrival time $X$. When $X$ has constant aging (i.e., $r_{X}(t)=c$ for some constant $c$, and hence memoryless), $I(t)$ becomes constant over time as well which in turn implies that the optimal sensing process should be periodic.

\section{Performance Evaluation}

\section{A. Dataset}

We use Wi-Fi AP contact logs of 84 iPhone 3G/3GS users and 60 Android users in KAIST campus. In case of iPhone measurement, users downloaded a measurement application and tested it for about 18 days in February 2010, recruited from an Korean iphone user community. The measurement application runs in the background to record Wi-Fi AP encounters every 3 minutes. We further measure the Wi-Fi contact logs of KAIST campus students for 14 days. We recruit 60 students with android smartphones and let them download a measurement application which logs Wi-Fi contacts with the granularity of 1 minute. In both measurements, the captured Wi-Fi APs include both private APs and commercial APs. The $\log$ files were periodically uploaded to a log-server over ftp connections. In addition, we also consider Active Hour (AH) scenarios of both traces, i.e., the traces from 9:00 to 23:00, because, in practice, many users may carry the mobile devices during active hours and charge them at night.

\section{B. Individual aging patterns}

We started with searching for distributions which fit best Inter-AP Arrival Time (IAT) and Contact Duration Time (CDT) of each individual. We selected three candidates of Weibull, Exponential and Generalized Pareto, because (i) Weibull and Exponential distributions [2], [13] are reported to follow IAT and/or CDT in literature, and (ii) Generalized Pareto significantly differ from Weibull and Exponential, possessing a power tail. We use a Cramer-Smirnov-Von-Mises (CSVM) statistical hypothesis test [30], [31] which is popularly used
TABLE II

$C S V M$ tests FOR THE DATASETS WITH SIGNIFICANCE LEVEL $=0.1 . \mathrm{EACH}$ ELEMENT $=\%$ OF ACCEPTED USERS ( $\%$ OF USERS WITH THE BEST MATCH)

\begin{tabular}{|l||c|c|c|}
\hline & Weibull & Gen. Pareto & Exp. \\
\hline \hline IAT: iPhone & $42(20)$ & $64(80)$ & $7(0)$ \\
\hline IAT: KAIST & $55(45)$ & $70(54)$ & $10(1)$ \\
\hline IAT: iPhone $(\mathrm{AH})$ & $54(18)$ & $68(79)$ & $22(3)$ \\
\hline IAT: KAIST $(\mathrm{AH})$ & $65(28)$ & $73(69)$ & $40(3)$ \\
\hline \hline CDT: iPhone & $48(28)$ & $52(68)$ & $13(4)$ \\
\hline CDT: KAIST & $64(42)$ & $56(53)$ & $30(5)$ \\
\hline CDT: iPhone $(\mathrm{AH})$ & $40(30)$ & $40(66)$ & $23(4)$ \\
\hline CDT: KAIST $(\mathrm{AH})$ & $68(32)$ & $67(60)$ & $43(8)$ \\
\hline
\end{tabular}

to find a best-fit. A CSVM test rejects a tested distribution when its CSVM statistic value is less than the Critical Values determined by a significance level. We test it with a popular significance level 0.1 and summarize the result of CSVM test in Table II. Each element in Table II consists of (i) \% of accepted users along with (ii) \% of users that have the best match with the corresponding distribution, which is in parentheses. We refer the readers to [30], [31] for the details of CSVM. Table II tells us that most users' IAT and CDT distributions follow Weibull or Generalized Pareto (Gen. Pareto), where Generalized Pareto's portion is larger.

It now remains to figure out the parameters of each distribution, i.e., $\alpha$ in Weibull and $\xi$ in Generalized Pareto, which shows the aging property of IAT and CDT. The actual value of $\alpha$ and $\xi$ for each individual will also be used in determining the actual sensing intervals of WiSAG. In Fig. 2(a) (resp. (b)), we plot the CDF of $\alpha$ for the users that experience a Weibull (resp. $\xi$ for Pareto) IAT distribution, demonstrating that about 94\% of overall users go through negative aging in their IAT distributions, i.e., $\alpha<1$ or $\xi>0$. We also plot the CDF of IATs during the Active Hours (AH), which also shows that $85 \%$ users have negative aging IAT distributions. Remarkably, all students in KAIST traces are perceived to undergo negative aging and most of them show heavy-tail distributions. However, when we focus on their IAT distributions during $\mathrm{AH}, 5 \%$ of students in KAIST appear to have positive aging. For the case of CDT distribution, the CDFs of $\alpha$ and $\xi$ are shown in Figs. 2(c) and (d). The shape parameter $\alpha$ of Weibull-fitted users ranges from 0.3 and 1.5 and $\xi$ of Pareto-fitted users are greater than -0.5 . These results support the fact that the sufficient condition (12) for the optimality can be highly likely to be satisfied (see Section III-D). 


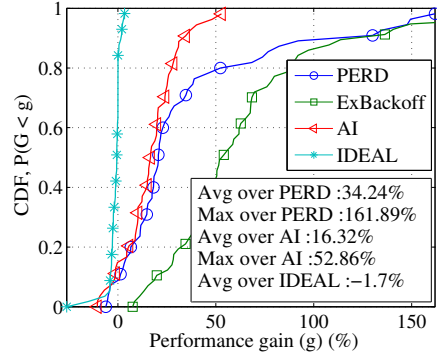

(a) iPhone

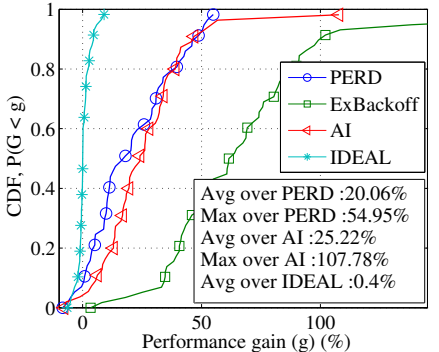

(b) iPhone, active hours

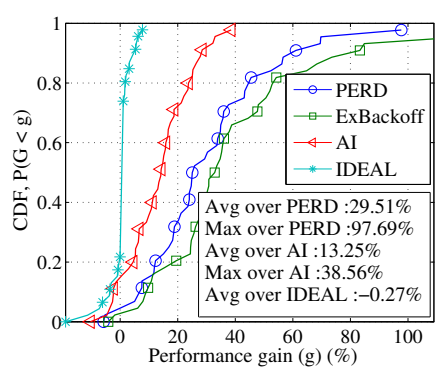

(c) KAIST (Android)

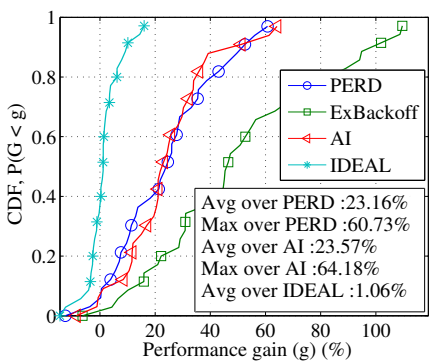

(d) KAIST (Android), active hours

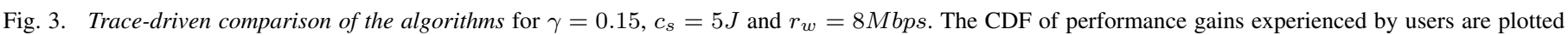

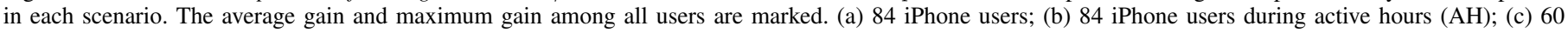
KAIST students (using Android smartphones); (d) 60 KAIST students during active hours (using Android smartphones).

The key message of our measurement-based analysis here is that a large portion of users experience negative aging, thereby implying that the sensing algorithms which increase the sensing intervals, such as AI [18], ExBackoff [32], and also WiSAG, take the right direction. However, just increasing intervals are not sufficient to guarantee high performance: the simple patterns of increasing intervals, such as linear in AI, or exponential in ExBackoff, do not always perform very well, and we need to adaptively control the sensing intervals like WiSAG that actively exploits the diverse failure rates of IAT over time. Further, the appearance of positive aging users during active hours indicate that, in some cases, algorithms with increasing interval, such as AI and ExBackoff, can perform poorly, whereas WiSAG can easily adapt to the user pattern with the decreasing sensing interval. We now verify such a reasoning through the trace-driven simulations.

\section{Tested algorithms}

We test three sensing algorithms in literature: PERD (Periodic) [13], [18] which senses APs over fixed periodic intervals, AI (Additive Increase) [18] which increases the sensing interval by a fixed increment after each sensing, and finally ExBackoff (exponential backoff) [32] which exponentially increases the sensing interval. Each algorithm has a parameter such as the period in PERD, the increment in AI, and the base in ExBackoff. In our comparison of the performance, we use the "best" version of each algorithm in the sense that the best parameter (which shows the minimum penalized cost) is searched by running the corresponding algorithm offline multiple times over a large number of parameter choices. In addition, we add one more algorithm IDEAL. Recall that a practical algorithm like WiSAG is unable to know whether the user misses an AP due to fast mobility or not. An omnipotent algorithm 'IDEAL' with the optimal sensing intervals can detect such AP missing events and initiate its sensing interval at the start of every phase (see the end of Section III-E for the discussion).

\section{Results}

We conduct trace-driven simulations based on the iPhone, KAIST campus traces. In our simulation, each individual mobile runs WiSAG with its own IAT and CDT distributions. By referring previous experimental measurements on the energy consumption and throughput of Wi-Fi [12], [16], we assume that a mobile consumes $5 J$ per a Wi-Fi sensing and an average throughput is $8 M b p s$. In Fig. 3, we plot the CDF of performance gains experienced by all users in each scenario against four algorithms. The performance gain over an algorithm ' $\mathrm{A}$ ' is defined as the increase of the penalized cost (in (OPT)) of ' $\mathrm{A}$ ' over that of WiSAG, $\left(\frac{\text { cost of ' } A \text { ' }- \text { cost of WiSAG }}{\text { cost of WiSAG }} \times 100\right)$. Fig. 3(a) shows that the average gains of iPhone users over PERD (resp. AI) is $34 \%$ (resp. 16\%). The maximum gain among all users is $161 \%$ over PERD and 52\% over AI. In the scenario of Fig. 3(b) where users carry their devices during active hours and charge them at night, WiSAG still outperforms other algorithm by $20 \%$ over PERD and $25 \%$ over AI on average. The maximum gain over PERD (resp. AI) is 54\% (resp. 107\%). The average performance gap between WiSAG and IDEAL is less than $1.7 \%$, which means that missing AP event due to fast mobility does not have huge effect on the performance. The average gains over ExBackoff for all cases are more than $65 \%$. Recall that PERD, AI, and ExBackoff operate with the best parameters in terms of our objective, acquired from offline computations over a large set of parameter candidates, whereas WiSAG computes the sensing intervals without any parameter tuning, relying only on the IAT and CDT distributions.

As shown in Fig. 3(c) of KAIST users, WiSAG outperforms PERD by $29.5 \%$ and AI by $13 \%$ on average. The maximum gain among all users are $97.7 \%$ over PERD and $38.6 \%$ over AI. A larger fraction of users with small $\alpha$ in Weibull and large $\xi$ in Pareto (thus negative aging), plotted in Figs. 2(a) and (b), leads to the worse performance in PERD and relatively better performance in AI. However, the active-hour scenario causes the poor performance of AI as shown in Fig 3(d), where the average gain over PERD (resp. AI) is 23\% (resp. 23.5\%). The maximum gains among all users during active hours are $60 \%$ over PERD and $64 \%$ over AI. We also observe as for such campus-wide trace that WiSAG performs closely to IDEAL and outperforms ExBackoff more than $40 \%$ on average.

The main reason behind the lower performance of AI during the active hours in both traces is that their shape parameters of IAT distributions tend to form light tails than those during all times, as already observed in Figs. 2(a) and (b). Moreover, due to the increase of the users with positive aging in their IAT distributions, the algorithms with increasing intervals (e.g., AI, ExBackoff) does not work well. To see this, we plot the average gain for IFR (positive aging) and DFR (negative aging) 


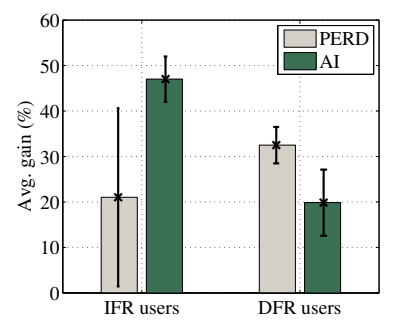

(a) Gains for IFR,DFR users

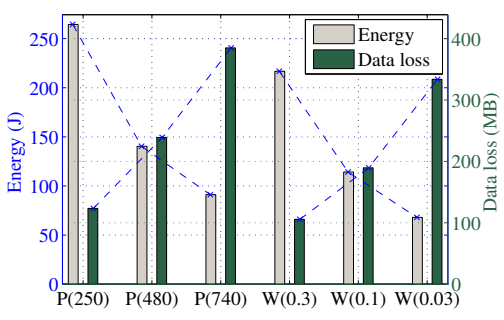

(b) Cost trade-off
Fig. 4. (a) The average performance gain of IFR (positive aging) and DFR (negative aging) users from both iPhone and KAIST traces during active hours. (b) The trade-off between the energy and data loss per a Wi-Fi contact in KAIST campus trace. In the x-axis, $P(s)$ and $W(r)$ means periodic sensing with fixed sensing interval $s(\mathrm{sec})$ and WiSAG with $\gamma=r$, respectively.

users in Fig 4. Using a CSVM test on two traces, we pick (i) IFR users whose IAT distributions during active hours are accepted to Weibull with $\alpha>1$ or Pareto with $\xi<0$, and (ii) DFR users who are fitted to Weibull with $\alpha<0.8$ or Pareto with $\xi>0$. For IFR users, WiSAG outperforms AI by $47 \%$ on average, since WiSAG successfully reduces the sensing intervals by adapting to the IFR distributions. For DFR users where the optimal sensing interval should increase over time, AI performs better than PERD. However, WiSAG still outperforms AI by $20 \%$, because the increasing intervals in WiSAG are more accurate. In Fig 4(b), we show that the tradeoff between energy and data loss in WiSAG can be controlled by $\gamma$. We observe that controlling the balance between the energy and data loss in periodic sensing is conducted on much higher cost region of both energy and data loss than WiSAG.

\section{CONCLUDING REMARKS}

The main contributions of this paper are two-folds. First, we analyze the fundamental interconnection between the tendency of "best" sensing intervals and distributions of inter-AP arrival and contact duration times. We classify these distributions by aging and show that the aging is the key property which decides whether to increase or decrease sensing intervals. Second, inspired by our analytical work, we develop a near-optimal Wi-Fi sensing algorithm, WiSAG, and show that WiSAG outperforms other algorithms through the extensive trace-driven simulations.

The study on Wi-Fi sensing algorithms includes the ones which use surrounding informations [13]-[16]. In some cases, such algorithms may outperform WiSAG which does not use the extra information. However, due to the additional overheads for using them, our focus is first to study the sensing patterns without surrounding informations by figuring out which factor is the key to the optimal blind sensing. We believe that WiSAG is widely beneficial in current smartphones, most of which also use a blind sensing policy [13]. It is left as a future work to extend our framework in WiSAG to account for the advantage of the surrounding information.

\section{ACKNOWLEDGMENT}

This work was supported by the Korea Communications Commission, under the R\&D program supervised by the Korea Communications Agency(KCA-2013-(11-911-05-004)), and was also supported in part by National Science Foundation under grants CNS-0831825 and CCF-0830680.

\section{REFERENCES}

[1] "Cisco visual networking index: Global mobile data traffic forecast update," feb 2011.

[2] K. Lee, I. Rhee, J. Lee, S. Chong, and Y. Yi, "Mobile data offloading: How much can wifi deliver?" in Proc. ACM CoNext, 2010.

[3] A. Balasubramanian, R. Mahajan, and A. Venkataramani, "Augmenting mobile 3g using wifi," in Proc. ACM Mobisys, 2010.

[4] M.-R. Ra, , J. Paek, A. B. Sharma, R. Govindan, M. H. Krieger, and M. J. Neely, "Energy-delay tradeoffs in smartphone applications," in Proc. ACM Mobisys, 2010.

[5] "Apple-icloud," 2011, http://www.apple.com/icloud/.

[6] "Microsoft pocket outlook," http://www.microsoft.com/windowsmobile/ en-us/downloads/microsoft/office-outlook-mobile.mspx.

[7] "Usc/enl. vcaps: Urban tomography project." http://tomography.usc.edu/.

[8] F. Baccelli and J. Bolot, "Modeling the economic value of the location data of mobile users," in Proc. IEEE Infocom, Apr. 2011.

[9] Devicespace, "Research on usage and trends," Wi-Fi Report, Jun. 2011.

[10] R. Friedman, A. Kogan, and Y. Krivolapov, "On power and throughput tradeoffs of wifi and bluetooth in smartphones," in Proc. IEEE INFOCOM, 2011

[11] A. Rahmati and L. Zhong, "Context-for-wireless: context-sensitive energy-efficient wireless data transfer," in Proc. ACM Mobisys, 2007.

[12] N. Balasubramanian, A. Balasubramanian, and A. Venkataramani, "Energy consumption in mobile phones: a measurement study and implications for network applications," in Proc. ACM IMC, 2009.

[13] K. Kim, A. Min, D. Gupta, P. Mohapatra, and J. Singh, "Improving energy efficiency of Wi-Fi sensing on smartphones," in Proc. IEEE Infocom, Apr. 2011.

[14] H. Wu, K. Tan, and J. Liu, "Footprint: cellular assisted wi-fi ap discovery on mobile phones for energy saving," in Proc. ACM WINTECH, 2009.

[15] R. Zhou, Y. Xiong, G. Xing, L. Sun, and J. Ma, "Zifi: wireless lan discovery via zigbee interference signatures," in Proc. ACM Mobicom, 2010.

[16] G. Ananthanarayanan and I. Stoica, "Blue-fi: Enhancing wi-fi performance using bluetooth signals," in Proc. ACM Mobisys, 2009.

[17] C. Research, "iPhone 4 owners tell us what they really think about their smart phones," ChangeWave Research Report, Aug. 2010.

[18] W. Wang, M. Motani, and V. Srinivasan, "Opportunistic energy-efficient contact probing in delay-tolerant applications," IEEE/ACM Transactions on Networking, vol. 17, pp. 1592-1605, 2009.

[19] C.-D. Lai and M. Xie, Stochastic Ageing and Dependence for Reliability. Springer, 2006.

[20] M. Kim and D. Kotz, "Periodic properties of user mobility and accesspoint popularity," Journal of Personal and Ubiquitous Computing, vol. 11, no. 6, pp. 465-479, Aug. 2007

[21] W. Hsu, T. Spyropoulos, K. Psounis, and A. Helmy, "Modeling timevariant user mobility in wireless mobile networks," in Proc. IEEE Infocom, 2007.

[22] H. Cai and D. Y. Eun, "Aging rules: What does the past tell about the future in mobile ad-hoc networks?" in Proc. ACM Mobihoc, 2009.

[23] N. Kaio and S. Osaki, "Comparison of inspection policies," The Journal of the Operational Research Society, vol. 40, pp. 499-503, 1989.

[24] R. E. Barlow, L. C. Hunter, and F. Proschan, "Optimum checking procedures," Journal of the Society for Industrial and Applied Mathematics, vol. 11, pp. 1078-1095, 1963.

[25] S. Kim and H. David, "On the dependence structure of order statistics and concomitants of order statistics," Journal of Statistical Planning and Inference, vol. 24, pp. 363-368, 1990.

[26] J.-Y. Le Boudec, Performance Evaluation of Computer and Communication Systems. Lasuanne, Switzerland: EPFL Press, 2010.

[27] I. Gelfand and S. Fomin, Calculus of Variations. Prentice-Hall, 1963.

[28] J. B. Keller, "Optimum checking schedulers for systems subject to random failure," Management Science, vol. 21, pp. 256-260, 1974.

[29] D. G. Luenberger, Optimization by Vector Space Methods. Wiley, 1969.

[30] V. Conan, J. Leguay, and T. Friedman, "Characterizing pairwise intercontact patterns in delay tolerant networks," in Proc. the 1st international conference on Autonomic computing and communication systems, 2007.

[31] W. T. Eadie, M. Roos, and F. James, Statistical Methods in Experimental Physics. Elsevier Sci. and and Tech., 1971.

[32] H. Falaki and S. Keshav, "Trace-based analysis of wi-fi scanning strategies," in Proc. ACM MobiCom Poster, 2008. 